\title{
A clinical study to evaluate the effects of adjunct yogic intervention on pulmonary functions in patients of bronchial asthma
}

\author{
Babita $^{1}$, Gulati K ${ }^{1 *}$, Ray A ${ }^{1}$, Menon BK ${ }^{2}$ and Rajkumar ${ }^{2}$ \\ ${ }^{1}$ Department of Pharmacology, Vallabhbhai Patel Chest Institute, University of Delhi, Delhi, India \\ ${ }^{2}$ Department of Pulmonary Medicine, Vallabhbhai Patel Chest Institute, University of Delhi, Delhi, India
}

\begin{abstract}
Purpose: The present study evaluated the effects of yogic intervention on pulmonary functions in the patients of bronchial asthma and compared with conventional pharmacotherapy for three months.

Method: Clinically diagnosed mild to moderate patients $(\mathrm{n}=100)$ of bronchial asthma were randomly assigned to 2 Groups. In Group I, patients were given conventional pharmacotherapy (inhaled corticosteroids + long acting beta-2 agonists) and Group II additionally received Yoga therapy for 3 months.

Results: Analysis of data showed significant improvement in pulmonary functions $\left(\mathrm{FEV}_{1}\right.$ and $\left.\mathrm{FEV}_{1} / \mathrm{FVC} \%\right)$ in Group I patients at1 month, however the effect was not persistent during $2^{\text {nd }}$ and $3^{\text {rd }}$ month. Interestingly, persistent significant improvement was observed in pulmonary functions in Group II patients at 1,2 and 3 months of treatment, those were given adjunct Yogic intervention with conventional pharmacotherapy.
\end{abstract}

Conclusion: The results suggested that Yoga as adjunct therapy enhanced the efficacy of conventional pharmacotherapy in patients of bronchial asthma.

\section{Introduction}

Bronchial asthma is a chronic inflammatory respiratory disease, characterized by complex interactions between inflammatory cells, mediators, and cytokines that result in airway constriction and airflow obstruction. The presence of airway edema and mucus secretion also contributes to airflow obstruction and bronchial hyperresponsiveness. The main objectives of asthma management are to achieve good symptoms control, diminish acute exacerbations and airflow limitation by use of pharmacotherapy with minimal side effects. The current pharmacotherapy depends on anti-inflammatory agents (corticosteroids, leukotriene inhibitors/antagonists etc.) and bronchodilators ( $\beta-2$ agonists, anticholinergics, methylxanthines etc.), but use of these drugs may also cause adverse effects thus resulting in poor compliance and incomplete treatment. The pharmacotherapy in asthmatics generally results in suppression of airway inflammation and reduction of bronchial hyperreactivity and airway obstruction. The short-term use of anti-asthma drugs are relatively safe, but chronic use may introduce many concerns not only regarding side effects but also drug insensitive/refractoriness. Corticosteroids are the mainstay in the pharmacotherapy and are widely used for the treatment of asthma, by virtue of their anti-inflammatory effects. However, the use of corticosteroids (inhalational or systemic) are associated with numerous side effects - the most common cause of adrenal insufficiency (systemic) and oral candidiasis (inhalation). Chronic use of corticosteroids inhibits the function of the hypothalamic-pituitary adrenal axis by negative feedback, which may cause adrenal in sufficiency which may persist even after the cessation of corticosteroid treatment [1,2]. Asthma patients have to depend on medicine lifelong to maintain their daily activities, and adverse effects of these drugs are a major reason for non-compliance and worsening of the disease. Thus, there is a need for devising alternative strategies for the safe and effective treatment of this condition. The use of Indian traditional medicinal concepts and products are being widely advocated by the Govt. of India and it's Ministry of AYUSH, because of their proven efficacy, safety, easy availability, and affordability. Further, interactions between traditional and modern systems of medicine are a subject matter of contemporary biomedical research. Accordingly, validation of traditional medicinal therapeutic regimens by using modern scientific methodology is also being encouraged and promoted. Traditional medicinal systems like Yoga could provide the answer for such situations in various chronic pathophysiological conditions, where the efficacy and safety of modern medications are inconsistent and unconvincing. Yoga, besides being a science, is an important way of life that has strongly emerged as alternative form of traditional therapy, particularly for chronic diseases, and the benefits are being acknowledged globally [3,4].

The present study evaluated the efficacy of daily yogic intervention for three months in the management of bronchial asthma by assessing the pulmonary functions of patients. Pulmonary function tests (PFT) are an important set of investigations used for monitoring lung functions of patients with respiratory pathology. It is a non-invasive

*Correspondence to: Kavita Gulati, Department of Pharmacology, Vallabhbhai Patel Chest Institute, University of Delhi, Delhi - 110007, India, E-mail: kavgul2002@yahoo.com

Received: December 12, 2018; Accepted: December 21, 2018; Published: December 28, 2018 
and valuable investigation in the diagnosis and management of patients with suspected or previously diagnosed respiratory disease [5]. Asthma is generally characterized by complete reversible airway obstruction, but it can also be partially irreversible, due to persistent inflammation. This, in turn, increases resistance to expiratory flow and may lead to decrease in forced respiratory volumes and flow rates [6,7]. Other parameters of the PFT include FVC (Forced Vital Capacity) and FEV1/ FVC \%. FVC is the total volume of air exhaled after a full inhalation and FEV1/FVC is the percentage of the vital capacity which is expired in the first second of maximal expiration. The FEV1 (volume of air expired in the first second during maximal expiratory effort) is the most widely used parameter to measure the mechanical properties of the lungs. It can be expressed as a percentage of the predictive value which allows classification of the severity of the impairment. The calculation of FEV1/FVC is a better index and allows the identification of obstructive or restrictive ventilatory defects. A FEV1/FVC $<70 \%$ where FEV1 is reduced more than FVC, signifies an obstructive defect [8].

\section{Material and method}

The present study was a randomized, open label, parallel design, controlled clinical study to evaluate the effects of adjunct Yoga therapy on pulmonary functions in patients of bronchial asthma, selected from the outpatient's department of the Vishwanathan Chest Hospital, Vallabhbhai Patel Chest Institute, Delhi. The study was carried out jointly by the Clinical Pharmacology Unit, Department of Pharmacology and Vishwanathan Chest Hospital, Vallabhbhai Patel Chest Institute. The study was initiated after obtaining the approval of the Institutional Ethical Committee and was conducted according to the ICH-GCP guidelines.

\section{Subjects}

In this study, total 100 patients of mild to moderate bronchial asthma from outdoor facilities were enrolled and randomly assigned to 2 Groups, after taking into consideration the laid down exclusion and inclusion criteria. After dropout of 15 patients, 5 patients from Group I and 10 patients from Group II, there were 45 patients in Group I (conventional therapy) and 40 patients in Group II (conventional therapy with Yoga therapy) in the study.

\section{Instruments}

Spirometry is the most reliable method to assess the lung functions, specifically the amount (volume) and/or speed (flow) of air that can be inhaled and exhaled. Computerized portable spirometer with ultrasonic flow head (Model: NDD Easy one PC Ultrasonic spirometer, USA) was used for the assessment. Spirometry is the most effective way of determining the severity, allowing calculation of $\%$ predicted FEV 1 and FVC from the resulting volume-time curve produced. The predicted values were calculated according to the age, height and weight of the patient and compared to the corresponding measured data, customize according to north Indian population with ethnic group correction to measure $\mathrm{FEV}_{1}$, FVC and FEV\%.

\section{Interventions}

In order to compare the extent of asthma control achieved after three months of respective treatment in two Groups i.e. a Group of patients on conventional pharmacotherapy and another Group with conventional pharmacotherapy and adjunct yogic intervention, PFT was done. Conventional therapy (Group I) consisted of patients on inhaled corticosteroids + long acting beta-2 agonists (ICS + LABA), whereas, Group II comprised of patients who were put on conventional therapy $(\mathrm{ICS}+\mathrm{LABA})+$ Yoga. Both Groups received SOS short acting beta-2 agonists as rescue medication.

\section{Statistical analysis}

Statistical analysis was carried out using the SPSS and graph pad prism (version 5.01) for Windows. The data are expressed as Mean \pm SEM and analysed by one-way ANOVA for repeated measures followed by post hoc Tukey's multiple comparison test and paired t-test variable appropriate. A minimum $P$ value $<0.05$ was used as a level of significance in all statistical tests.

\section{Results}

\section{Socio-demographic data}

Analysis of the data showed that there was no significant difference in the age of the patients in both groups. The male: female sex ratio for Group I was 21:24 and Group II was 23:17. The mean age for Group I was $36 \pm 11.99$ and Group II was $32 \pm 10.33$. There was no significant difference found statistically in both age and sex distribution, both groups were also comparable at baseline with respect to severity of asthma. The asthma severity results are summarized in Table 1.

\section{Pulmonary functions test}

Analysis of data showed that pulmonary functions $\left(\mathrm{FEV}_{1}\right.$ and $\mathrm{FEV}_{1} / \mathrm{FVC} \%$ ) improved in both the Groups but persistently significant improvement was observed in Group II patients only for 3 months. The results are shown in Table 2 and Figure 1.

\section{Discussion}

In this study, results showed that $\mathrm{FEV}_{1}$ values were improved in both the Groups after treatments, but persistent and remarkable percentage increases were observed in Group II as compared to Group I. Group I patients showed significant improvement in FEV $_{1}$ after 1 month of pharmacotherapy, but surprisingly the percentage improvement in $\mathrm{FEV}_{1}$ decreased, after $2^{\text {nd }}$ and $3^{\text {rd }}$ months. Similar findings were observed by Aggarwal et al. [9] who reported improvement in PFT parameters after 1 month of treatment but not in 2 and 3 months in control Group (conventional pharmacotherapy). This can be due to alteration in cortisol

Table 1. Distribution of patients as per asthma severity in Group I and Group II

\begin{tabular}{|c|c|c|c|c|}
\hline \multirow{2}{*}{$\begin{array}{c}\text { Bronchial } \\
\text { Asthma }\end{array}$} & \multicolumn{2}{|c|}{$\begin{array}{c}\text { Group I } \\
\text { (45) }\end{array}$} & \multicolumn{2}{c|}{$\begin{array}{c}\text { Group II } \\
\text { (40) }\end{array}$} \\
\cline { 2 - 5 } & No. & \% & No. & \% \\
\hline Mild & 34 & 75.6 & 28 & 70.0 \\
\hline Moderate & 11 & 24.4 & 12 & 30 \\
\hline Total & 45 & 100 & 40 & 100 \\
\hline
\end{tabular}

Sample are matched according to severity of asthma, $\mathrm{N}=45$ in Group I and N=40 in Group II.

Table 2. Comparison of pulmonary functions of the patients of bronchial asthma of Group I (conventional treatment) and Group II (conventional treatment with Yoga therapy)

\begin{tabular}{|c|c|c|c|c|c|}
\hline Parameters & Groups & Baseline & 1 month & 2 month & 3 month \\
\hline \multirow{2}{*}{$\mathrm{FEV}_{1}(\mathrm{~L})$} & Group I & $2.247 \pm 0.12$ & $2.539 \pm 0.30 *$ & $2.253 \pm 0.16$ & $2.416 \pm 0.11$ \\
\hline & Group II & $2.196 \pm 0.09$ & $2.506 \pm 0.13 *$ & $2.658 \pm 0.15 *$ & $2.701 \pm 0.12 * *$ \\
\hline \multirow{2}{*}{$\mathrm{FVC}(\mathrm{L})$} & Group I & $3.096 \pm 0.12$ & $3.333 \pm 0.48$ & $3.032 \pm 0.13$ & $3.034 \pm 0.12$ \\
\hline & Group II & $3.208 \pm 0.15$ & $3.582 \pm 0.17$ & $3.304 \pm 0.19$ & $3.299 \pm 0.16$ \\
\hline \multirow{2}{*}{$\begin{array}{c}\mathrm{FEV}_{1} / \mathrm{FVC} \\
(\%)\end{array}$} & Group I & $73.53 \pm 1.94$ & $78.37 \pm 2.65 *$ & $76.87 \pm 3.30$ & $77.23 \pm 1.48$ \\
\hline & Group II & $71.42 \pm 1.81$ & $74.88 \pm 2.07 *$ & $76.18 \pm 2.04 *$ & $80.00 \pm 1.31 * *$ \\
\hline
\end{tabular}

All data are expressed as mean \pm SEM. ${ }^{*} \mathrm{p}<0.05, * * \mathrm{p}<0.01$ based on paired $\mathrm{t}$ test comparisons with baseline values at 0 month. 


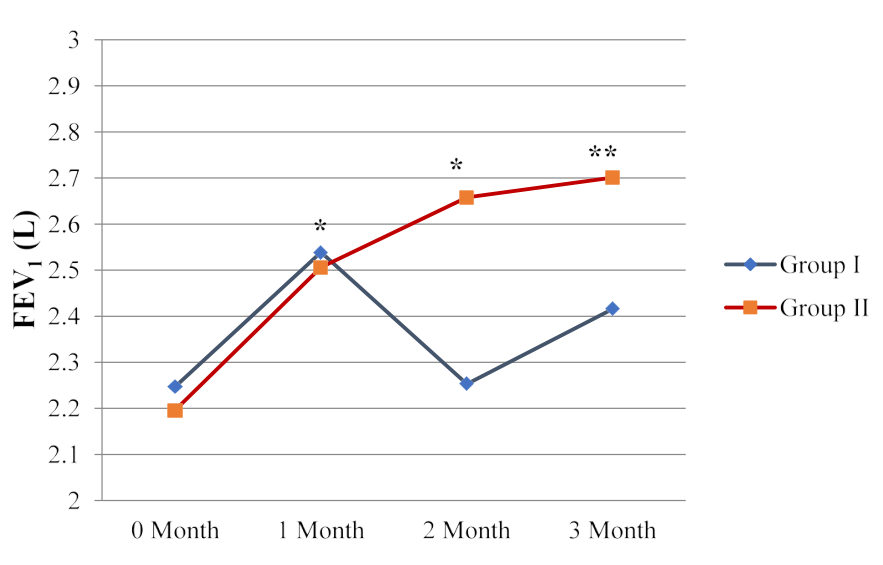

Figure 1. Comparison of $\mathrm{FEV}_{1}(\mathrm{~L})$ between Group I (conventional treatment) and Group II

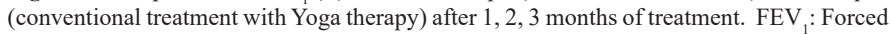
expiratory volume in 1 second.

${ }^{*} \mathrm{p}<0.05, * * \mathrm{p}<0.01$ based on paired $\mathrm{t}$ test comparisons with baseline values at 0 month.

level by treatment with corticosteroids for three months. It has been reported that administration of exogenous glucocorticoids (oral, inhalational) at high doses for prolonged periods and even small doses for only few days may cause ACTH suppression and downregulation of hypothalamic-pituitary-adrenal (HPA) axis. As a result, in the absence of ACTH, the adrenal cortex may lose the ability to produce cortisol $[10,11,12]$. Cortisol is a steroid hormone in the body and its level is shown to be correlated with inflammation i.e. lowered levels of cortisol may increase inflammation in the airways [13]. Earlier, Dwyer et al. [14] also suggested that steroid treatment in asthmatic patients may result in a low plasma cortisol response, which may lead to refractoriness and poor clinical improvement. Interestingly, the Group II patients, who received adjunct yogic intervention, showed significant, persistent improvement in $\mathrm{FEV}_{1}$ throughout the period of follow up i.e. for three months, thus suggesting greater reduction in airway obstruction and persistent control of disease. This may be due to the fact that yogic intervention could have maintained normal cortisol's level in Group II patients which may have resulted in persistent improvement in lung function, as has also been reported by Curtis et al. [15]. This is also supported by Bhavanani [16] who showed that the practice of Yoga maintains homeostasis of body through improved balancing and functioning of the psycho-immuno-neuro-endocrine system. However, further studies determining the level of cortisol in a larger group of patients will be required to strengthen the hypothesis.

The present results of PFT showed that in Groups II (adjunct Yoga Group), the FVC values improved from that of baseline values with the Yoga + conventional drug treatment at the end of $1^{\text {st }}$ month, which remained persistent for three months, whereas in Group I improvement in FVC values was not consistent for three months. However, both groups showed statistically insignificant data from baseline after 3 months' of respective treatments. Although, FVC, a measure of lung volume, is usually decreased in diseases that reduce the lungs expansion, reduced FVC can occur despite a normal or increased total lung volume and may not be a reliable indicator of total lung capacity or restriction, especially in the setting of airflow obstruction [8].

The $\mathrm{FEV}_{1} / \mathrm{FVC}$ is the percentage of the vital capacity which is expired in the first second of maximal expiration. The present result showed that $\mathrm{FEV}_{1} / \mathrm{FVC}$, values were improved in both groups after treatments, but persistent and remarkable percentage increase were observed in Group
II as compared to Group I. Group I showed maximal improvement in $\mathrm{FEV}_{1} / \mathrm{FVC}$ after 1 months of conventional treatment which declined after $2^{\text {nd }}$ and $3^{\text {rd }}$ months of treatment. The trend of results is similar to that observed with $\mathrm{FEV}_{1}$ and may be due to alteration in the levels of endogenous cortisol after corticosteroid treatment. However, in Group II statistically significant and persistently increases in $\mathrm{FEV}_{1} / \mathrm{FVC} \%$ was observed at the end of each month. This suggested greater reduction in airway obstruction in Group II patients, i.e. those who received conventional pharmacotherapy + adjunct Yogic intervention.

Our results are supported by the previous findings of Sodhi et al. [17], who reported that 2 months of Yoga as an adjunct therapy significantly improved pulmonary functions in bronchial asthma patients. Nagarthna and Nagendra [18] also showed that peak flow rate was improved after 54 months of yogic intervention in patients of bronchial asthma. The results of the present study are comparable to that of Vempati et al. [19] which showed significant, steady and progressive improvement in $\mathrm{FEV}_{1}$ and $\mathrm{FEV}_{1} / \mathrm{FVC} \%$ in the Yoga Group but not in the control Group - thus indicating the efficacy of Yoga. Singh et al. [20] also reported statistically significant improvement in FEV and $\mathrm{FEV}_{1} / \mathrm{FVC} \%$ of lung function after 2 months of Yoga practices + life style modification, which included a diet chart and sleeping pattern. The results are comparable to our findings, although, in the present study, only a fixed protocol of Yoga was given without any life style modification. Similarly, Biju et al. [21] showed significant improvements in $\mathrm{FEV}_{1}$ and $\mathrm{FEV}_{1} / \mathrm{FVC} \%$ in the case Group as compared to control Group whereas no significant change occurred in FVC. Harika et al. [22] also showed statistically significant improvement in $\mathrm{FEV}_{1}, \mathrm{FVC}$ but not in $\mathrm{FEV}_{1} / \mathrm{FVC} \%$. Agnihotri et al. [23] reported improvement in pulmonary function and concluded that Yoga as an adjuvant therapy to standard medical therapy may be an effective alternative in the asthma management.

\section{Conclusion}

Pharmacotherapy with corticosteroids is highly effective treatment in bronchial asthma because of anti-inflammatory activity but in long-term use may cause alteration in cortisol level which may result in poor clinical improvement. It can be concluded that Yoga as an adjunct therapy in patients of bronchial asthma showed persistent improvement in pulmonary functions, this may be due to maintenance of homeostatic balance of body.

\section{Acknowledgement}

The financial support from Ministry of AYUSH is gratefully acknowledged for conducting this research work. We are also thankful to Mr. Manoj and the team from Morarji Desai National Institute of Yoga (MDNIY), Govt. of India for providing yoga training as per the study protocol to the patients of asthma.

\section{References}

1. Broersen LHA, Pereira AM, Jorgensen JOL, Dekkers OM (2015) Adrenal Insufficiency in Corticosteroids Use: Systematic Review and Meta-Analysis. J Clin Endocrinol Metab 100: 2171-2180.

2. Bondugulapati LNR and Rees DA (2016) Inhaled corticosteroids and HPA axis suppression: how important is it and how should it be managed? Clin Endocrinol 85: 165-169.

3. Hoyez AC (2007) The 'world of yoga': the production and reproduction of therapeutic landscapes. Soc Sci Med 65: 112-124. [Crossref]

4. Raghuwanshi A (2011) A review: History of revival of yoga in 20th century and establishment of yoga as a science in 21st century. Int SCi Yoga J SENSE 1: 208-216. 
5. Madan D, Singal P, Kaur H (2010) Spirometric evaluation of pulmonary function tests in bronchial asthma patients. JESP 6: 106-111.

6. Boulet LP (2009) Irreversible airway obstruction in asthma. Curr Allergy Asthma Rep 9: $168-173$.

7. Cibella F, Cuttitta G, Bellia V, Bucchieri S, D'Anna S, et al. (2002) Lung function decline in bronchial asthma. Chest 122: 1944-1948. [Crossref]

8. Ranu H, Wilde M, Madden B (2011) Pulmonary function tests. Ulster Med J 80: 84-90. [Crossref]

9. Aggrawal T, Khatri A, Sidique SS, Hasan SN, Deepankar, Kulshreshtha M, et al. (2013) Pranayama has additive beneficial effects along with medication in bronchial asthma patients. J Phys Pharm Adv 3: 292-297.

10. Priftis K, Milner AD, Conway E, Honour JW (1990) Adrenal function in asthma. Arch Dis Child 65: 838-840. [Crossref]

11. Todd GRG, Acerini CL, Ross-Russell R, S Zahra S, Warner JT, (2002) Survey of adrenal crisis associated with inhaled corticosteroids in the United Kingdom. Arch Dis Child 87: 457-461.

12. Ahmet A, Kim H, Spier S (2011) Adrenal suppression: A practical guide to the screening and management of this under-recognized complication of inhaled corticosteroid therapy. Allergy Asthma Clin Immunol 7: 1-12. [Crossref]

13. Chen E, Miller GE (2007) Stress and inflammation in exacerbations of asthma. Brain Behav Immun 21: 993-999. [Crossref]

14. Dwyer J, Lazarus L, Hickie JB (1967) A study of cortisol metabolism in patients with chronic asthma. Inter Med J 16: 298-304.
15. Curtis K, Osadchuk A, Katz J (2011) An eight-week yoga intervention is associated with improvements in pain, psychological functioning and mindfulness, and changes in cortisol levels in women with fibromyalgia. J Pain Res 4: 189-201. [Crossref]

16. Bhavanani AB (2011) Understanding the science of yoga. IJoY 1: 334-344.

17. Sodhi C, Singh S, Dandona PK (2009) A study of the effect of yoga training on pulmonary functions in patients with bronchial asthma. Indian J Physiol Pharmacol 53: 169-174. [Crossref]

18. Nagarathna R, Nagendra HR (1985) Yoga for bronchial asthma: a controlled study. $B r$ Med J (Clin Res Ed) 291: 1077-1079. [Crossref]

19. Vempati R, Bijlani RL, Deepak KK (2009) The efficacy of a comprehensive lifestyle modification programm based on yoga in the management of bronchial asthma: a randomized controlled trial. BMC Pulm Med 9: 37

20. Singh S, Soni R, Singh KP, Tandon OP (2012) Effect of yoga practices on pulmonary function tests including transfer factor of lung for carbon monoxide (TLCO) in asthma patients. Indian J Physiol Pharmacol 56: 63-68. [Crossref]

21. Biju B, Geetha N, Sobhakumari T (2012) Yoga training with meditation ameliorate the asthmatic attack by improving pulmonary functions: a pilot study. Nat J Med Res 2: $182-187$.

22. Harika P, Sayyad R, Yadav PK, Sah S, Kar SK (2014) Evaluation of pulmonary function test before and after yoga practice in asthma patients. IJBAMR 3: 8-14.

23. Agnihotri S, Kant S, Mishra SK, Tripathi PM (2015) Role of yoga in asthma management. HDD 2015; 2: ISSN 2382-1019.

Copyright: (C)2018 Babita. This is an open-access article distributed under the terms of the Creative Commons Attribution License, which permits unrestricted use, distribution, and reproduction in any medium, provided the original author and source are credited. 